\title{
The role of quarantine ward in the COVID-era: a Taiwan medical center experience
}

\author{
Tse-Hao Chen ${ }^{1} \wedge$, Wei-Yang Chi ${ }^{1} \wedge$, Shih-Yi Yang ${ }^{1}$, Chen-Hao Liao ${ }^{1}$, Yung-Lun Tsai ${ }^{1}$, \\ Wen-Han Chang ${ }^{1,2,3,4}$, Weide Tsai ${ }^{1,2,3} \wedge$
}

${ }^{1}$ Department of Emergency Medicine, Mackay Memorial Hospital, Taipei, Taiwan; ${ }^{2}$ Department of Medicine, Mackay Medical College, New Taipei City, Taiwan; Hospital, Taipei, Taiwan; ${ }^{3}$ Mackay Medicine, Nursing and Management College, Taipei, Taiwan; ${ }^{4}$ Institute of Mechatronic Engineering, National Taipei University of Technology, Taipei, Taiwan

Contributions: (I) Conception and design: TH Chen, WH Chang, W Tsai; (II) Administrative support: WH Chang, W Tsai; (III) Provision of study materials or patients: SY Yang; (IV) Collection and assembly of data: CH Liao; (V) Data analysis and interpretation: WY Chi, YL Tsai; (VI) Manuscript writing: All authors; (VII) Final approval of manuscript: All authors.

Correspondence to: Weide Tsai. No. 92, Sec. 2, Zhongshan N. Rd., Taipei City 10449, Taiwan. Email: weidetsai@gmail.com.

\begin{abstract}
The coronavirus disease 2019 (COVID-19) has become a global pandemic since March 2020 and caused healthcare insufficient resources, economic and political issues. Taiwan was one of the few successful countries to contain the vicious virus without community spread. Since the Severe Acute Respiratory Syndrome (SARS) outbreak in 2013, quarantine wards have been proposed for controlling the possible highly contagious disease. In the COVID-19 era, MacKay Memorial Hospital in Taiwan had been operating quarantine rooms since April 2020. Patients who needed hospitalization for fever, COVID-like symptoms, or pneumonia were admitted to quarantine rooms while waiting for the result of the COVID-19 reverse transcriptase-polymerase chain reaction (RT-PCR) test. We implemented a well-established quarantine policy without visitors allowed during the patient's quarantine period. Healthcare workers must self-report fever or COVID-like symptoms every day. We started the release evaluation from quarantine after receiving the negative result of COVID-19 RT-PCR. Infection disease (ID) physicians were in charge of the release process. If a patient continued to have any persistent COVID-like symptoms or uncontrolled pneumonia, ID physicians would not grant the release from quarantine. Since the establishment of the quarantine rooms, over one thousand suspected COVID-19 patients had been admitted. There has been no community transmitted cases reported for more than 200 days as of the end of November 2020 in Taiwan. Good practice of preventive measures like our operation of quarantine wards is early and vital planning of the hospital strategy against the spread of COVID-19.
\end{abstract}

Keywords: Coronavirus disease 2019 (COVID-19); quarantine ward; Taiwan; infection control

Received: 24 December 2020; Accepted: 13 April 2021; Published: 18 May 2021.

doi: $10.21037 /$ ht-20-35

View this article at: http://dx.doi.org/10.21037/ht-20-35

\section{Introduction}

On January 21st 2020, Taiwan faced the first coronavirus disease 2019 (COVID-19) patient. Since then, more than six hundred confirmed cases encountered in our country.
However, Taiwan was one of the most successful countries to contain the notorious virus without community spread.

World Health Organization (WHO) issued COVID-19 as a pandemic on March 11th, 2020. There was a significant increase in confirmed patient volume since then. The

^ ORCID: Tse-Hao Chen 0000-0002-6370-7068; Wei-Yang Chi 0000-0001-5295-156X; Wen-Han Chang 0000-0002-2071-7258; Weide Tsai 00000001-7859-2032. 
impact of COVID-19 was heavily on the healthcare sector and the parts of the lifestyle, economy, and politics. Focus on prevention and management of COVID-19 should be the priority of healthcare staff.

MacKay Memorial Hospital is a medical center level institution in the crowded Taipei city. It has an annual emergency room (ER) visits of 130,000 patients ranking No.2 busiest ER in Taiwan. The quarantine ward is a crucial measure for managing the suspected COVID-19 patients in a free of local transmission case country. We re-opened eight quarantine rooms on April 14th, 2020, and would like to share our experience of establishing and managing a quarantine ward in this cruel COVID era.

\section{Need for establishing quarantine ward}

Precise diagnosis of COVID-19 relies on either reverse transcriptase-polymerase chain reaction (RT-PCR) or chest computer tomography (CT). In Taiwan, RT-PCR is preferred over routine chest CT examination. A previous study supported this policy (1). A meta-analysis compared the chest CT's diagnostic value and RT-PCR revealed pooled chest CT sensitivity was higher than RT-PCR (94\% vs. 89\%). However, chest CT's low specificity led to both positive predictive value and negative predictive value of CT inferior to RT-PCR (1). As a result, RT-PCR was recommended as a diagnostic tool of COVID-19 in a low-prevalence rate country $(1,2)$. Nonetheless, this "gold standard" examination takes hours to complete (3). The need for a quarantine ward for newly admitted patients while waiting for RT-PCR testing result was crucial to prevent possible in-hospital spread.

The analysis of severe acute respiratory syndrome (SARS) in-hospital outbreak indicated that bed distance lesser than one meter and performing resuscitation would significantly elevate the risk of SARS in-hospital spread (4). Massive patients visit and crowding of the emergency room led to such a dangerous environment, so the setup of a quarantine ward was inevitable. A former study utilizing a mathematical model of COVID-19 transmission revealed encouraging results of early testing and monitoring in the quarantine ward (5). A 2,500-bed tertiary hospital in South Korea found that both early RT-PCR testing within 8 hours and the monitoring in quarantine ward models for newly admitted patients could decrease the in-hospital COVID-19 infection rates $81.3 \%$ and $70 \%$, respectively (5). Calling for a quarantine ward to separate patients with possible
COVID-19 infection was proposed worldwide (6-8).

\section{Quarantine policy}

The quarantine ward consists of eight triple rooms in single-use; each room accommodates a patient with or without a family or caregiver. The patient and family or caregiver cannot leave the quarantine ward before the RT-PCR test result becomes available, and it is at the ID physician's discretion to order a de-isolation when the result is negative. No visitors are permitted in the quarantine ward. Quarantined patients are educated to report instantly any new-onset symptoms when admitted.

Quarantine ward working staff are different from ones in the ordinary ward and the emergency room. Staff practice fever or COVID-19 like symptoms self-screening before work. If fever or COVID-19 like symptoms present, staff take sick leave and receive COVID-19 RT-PCR test. This policy aims to reduce the possible hospital-acquired spread of COVID-19. Essential personal protective equipment (PPE), including surgical gloves, barrier gown, and face shield, is provided routinely to the staff as a priority. The hospital mandates N95 face mask fitting exercise to all staff. After the patient care, staff disposes of face mask, surgical gloves, and barrier gown, any reusable equipment used during the care go through the disinfection process using 1,000 ppm diluted sodium dichloroisocyanurate. Strict handwashing protocol before and after patient care is enforced. A study of healthcare workers undressed PPE in a designated semi-contaminated area, where partitions were used between the quarantine ward and clean area (9), revealed that the COVID-19 virus was not detected in the semi-contaminated zone where patients had no access. This finding could support our strategy that physical barriers were an effective method to prevent viral spread.

A previous study also supported our bundle of infection prevention measures in the quarantine ward (10). Use of PPE and surgical mask during procedures, hand hygiene among healthcare workers and suspected patients could keep the hospital from the nosocomial transmission of COVID-19 in a Hong Kong hospital treating 42 COVID-19 patients.

Another crucial issue was the mental health of patients and quarantine healthcare workers. Studies indicated that quarantined patients could suffer from post-traumatic stress symptoms or general mental health problems even after the quarantine period $(11,12)$. Some actions were suggested, 
such as providing sufficient information, providing adequate supplies, and reducing boredom (12). Our quarantine ward staff included social workers and psychologists. Whenever patients were in a distressed mood, timely mental support could lift patients through this gloom process. Our quarantine ward social workers and psychologists also took care of healthcare workers' mental health. Closely monitor staff's mental pressure could prevent them from burning out in a short-handed situation. A study also pointed out that support from the management echelon could help healthcare workers' mental protection (12). Moreover, managers should also ensure sufficient human resources for the quarantine wards.

\section{Admission criteria}

Patients presented to our emergency department with one of the following findings, body temperature higher than $38^{\circ} \mathrm{C}$, upper respiratory symptoms and positive to the history of oversea travel, certain occupations, contact, or cluster (TOCC) were rerouted to the screening station. Separating high-risk patients was the first step of our infection control policy in the emergency room. Patients who underwent workups and required hospitalization were obligated to receive COVID-19 RT-PCR testing before admission. Moreover, patients who needed hospitalization with COVID-like symptoms, abnormality in taste or smell, and pneumonia were also admitted to the quarantine ward during the RT-PCR test in the process.

Patients transferred to the quarantine ward required an acrylic shield over the head to decrease airborne transmission. A designated elevator was used for the transfer of quarantine ward patients. We disinfected the transfer route, including the elevator from the ER to the quarantine ward, with chlorine dioxide mist spray after each transfer.

\section{Release from quarantine}

Quarantine release of suspected COVID-19 patients remained a challenge for ID physicians globally (13). The previous report addressed by Singapore's National Centre for Infectious Diseases proposed that patients met the following criteria, afebrile for one day, two sets of negative COVID RT-PCR result, the day of symptoms onset was at least six days earlier, and other respiratory pathogens detected; can be released from quarantine into an ordinary ward or discharged (13).

Most of the COVID-19 cases were imported in Taiwan.
We released suspected COVID patients from quarantine after one set of negative RT-PCR results. The infection disease physician was in charge of this process. If any COVID-19 like symptoms persisted or uncontrolled pneumonia encountered, quarantine release would not be granted.

The disinfection procedure started after the patient had quarantine release and left the quarantine room. A non-use period of at least 60 minutes was required before the janitor started disinfection. The reason for this policy was the possible aerosol transmission of COVID-19 $(9,14,15)$. After this non-use period, janitors must wear PPE and clean all surfaces in the quarantine room with $5,000 \mathrm{ppm}(0.5 \% ; 1: 10)$ of sodium dichloroisocyanurate or bleach. The disinfection process was repeated a second time with an interval of 60 minutes. A former study demonstrated that the floor, table, chair, and bed where patients frequently contacted showed intense viral load (14). Another study also pointed out that medical equipment, touch screens, shelves, door handles, and bedrails were mostly contaminated after COVID-19 patients' use (9). Our janitors were trained to pay special attention to where patients had frequent direct physical contact.

\section{Current status}

From April 14th to October 30th, 511 patients were admitted to the quarantine room. Average quarantine time was 35.8 hours, with the longest 92 hours and the shortest 14 hours. $46.5 \%$ of the released patients were transferred to the infection ward, followed by $18.4 \%$ of the patients transferred to the chest ward. $10.5 \%$ of the patients were released from quarantine and discharged simultaneously.

13 COVID-19 patients were diagnosed in MacKay Memorial Hospital emergency department and hospitalized. There was no in-hospital transmission of COVID-19 as of the end of November 2020.

\section{Preparing for mass casualty incident}

Although Taiwan was one of the most successful countries to close down COVID-19, the potential mass casualty incident cannot be ignored. The uncertainty of developing an effective COVID-19 vaccine also warns us of the possibility of another episode of a pandemic attack. Since quarantine wards were originally triple rooms, a possible triple room in double use with spacing more than 1-meter distance can be established. Our strategy was to make the 
best use of quarantine ward space at the beginning of the mass casualty incident. A report from Daegu Dongsan Hospital of Keimyung University also approved this strategy (16). By distributing 666 confirmed COVID-19 patients into two types of rooms, a double room in single use or double room, patients were observed for 14 days. RT-PCR negative conversion rate, symptoms improvement rate, and time to de-effervescence revealed no statistical difference (16). However, a further plan for massive patients should be established.

\section{Conclusion}

In the devastating COVID-19 era, establishing of quarantine ward was necessary. Crowding of the emergency room was a risk factor of the possible in-hospital spread of COVID-19. Quarantine of possible COVID-19 patients, while RT-PCR test was in the process, was a good act. Strict enforcement of quarantine policy was an essential factor to avoid the in-hospital spread of COVID-19.

\section{Acknowledgments}

Funding: None.

\section{Footnote}

Conflicts of Interest: All authors have completed the ICMJE uniform disclosure form (available at http://dx.doi. org/10.21037/ht-20-35). WHC serves as an unpaid Editorin-Chief of Health Technology. The authors have no other conflicts of interest to declare.

Ethical Statement: The authors are accountable for all aspects of the work in ensuring that questions related to the accuracy or integrity of any part of the work are appropriately investigated and resolved.

Open Access Statement: This is an Open Access article distributed in accordance with the Creative Commons Attribution-NonCommercial-NoDerivs 4.0 International License (CC BY-NC-ND 4.0), which permits the noncommercial replication and distribution of the article with the strict proviso that no changes or edits are made and the original work is properly cited (including links to both the formal publication through the relevant DOI and the license). See: https://creativecommons.org/licenses/by-nc$\mathrm{nd} / 4.0 /$.

\section{References}

1. Kim H, Hong H, Yoon SH. Diagnostic Performance of CT and Reverse Transcriptase-Polymerase Chain Reaction for Coronavirus Disease 2019: A Meta-Analysis. Radiology 2020;296:E145-E155.

2. Zitek T. The Appropriate Use of Testing for COVID-19. West J Emerg Med 2020;21:470-2.

3. Esbin MN, Whitney ON, Chong S, et al. Overcoming the bottleneck to widespread testing: a rapid review of nucleic acid testing approaches for COVID-19 detection. RNA 2020;26:771-83.

4. Yu IT, Xie ZH, Tsoi KK, et al. Why did outbreaks of severe acute respiratory syndrome occur in some hospital wards but not in others? Clin Infect Dis 2007;44:1017-25.

5. Baek YJ, Lee T, Cho Y, et al. A mathematical model of COVID-19 transmission in a tertiary hospital and assessment of the effects of different intervention strategies. PLoS One 2020;15:e0241169.

6. Wee LEI, Sim XYJ, Conceicao EP, et al. Containing COVID-19 outside the isolation ward: The impact of an infection control bundle on environmental contamination and transmission in a cohort general ward. Am J Infect Control 2020;48:1056-61.

7. Bo M, Brunetti E, Presta R, et al. To keep a COVID-19free hospital ward: mission possible? Aging Clin Exp Res 2020;32:1627-8.

8. Lee FC, Wee WK, Johan A. Public hospital preparations for SARS outbreak: experience of Alexandra Hospital. Prehosp Disaster Med 2005;20:24-31.

9. Razzini K, Castrica M, Menchetti L, et al. SARSCoV-2 RNA detection in the air and on surfaces in the COVID-19 ward of a hospital in Milan, Italy. Sci Total Environ 2020;742:140540.

10. Cheng VCC, Wong SC, Chen JHK, et al. Escalating infection control response to the rapidly evolving epidemiology of the coronavirus disease 2019 (COVID-19) due to SARS-CoV-2 in Hong Kong. Infect Control Hosp Epidemiol 2020;41:493-8.

11. Modini M, Vrklevski L. A hotel room on Mars: quarantine and the psychological view from the virtual front line. Australas Psychiatry 2020;28:624-6.

12. Brooks SK, Webster RK, Smith LE, et al. The psychological impact of quarantine and how to reduce it: rapid review of the evidence. Lancet 2020;395:912-20.

13. Tay JY, Lim PL, Marimuthu K, et al. De-isolating Coronavirus Disease 2019 Suspected Cases: A Continuing Challenge. Clin Infect Dis 2020;71:883-4. 
14. Ong SWX, Tan YK, Chia PY, et al. Air, Surface Environmental, and Personal Protective Equipment Contamination by Severe Acute Respiratory Syndrome Coronavirus 2 (SARS-CoV-2) From a Symptomatic Patient. JAMA. 2020;323:1610-1612.

15. Anderson EL, Turnham P, Griffin JR, et al. Consideration

doi: 10.21037/ht-20-35

Cite this article as: Chen TH, Chi WY, Yang SY, Liao CH, Tsai YL, Chang WH, Tsai W. The role of quarantine ward in the COVID-era: a Taiwan medical center experience. Health Technol 2021;5:9. of the Aerosol Transmission for COVID-19 and Public Health. Risk Anal 2020;40:902-7.

16. Hyun M, Lee JY, Kwon YS, et al. COVID-19: Comparing the applicability of shared room and single room occupancy. Transbound Emerg Dis 2020. [Epub ahead of print]. doi: $10.1111 /$ tbed.13853. 